\title{
Introduction
}

\section{Agitation in brain injury}

Perhaps one of the most controversial and poorly understood phenomena in brain injury care and research is agitation. There is little consensus about this entity within the field of physical medicine and rehabilitation, let alone consensus among neuroscience professionals. The lack of agreement is so basic that use of the term agitation is questioned. This lack of consensus was the genesis of this issue of NeuroRehabilitation.

Articles approach the phenomenon of brain injury agitation from several disciplines, spanning assessment to new frontiers in neuropharmacology. This variety of perspectives is both healthy and necessary to assist the characterization of agitation following brain injury. It challenges disciplines to rethink the neurobehavioral sequelae of brain injury and hopefully will stimulate further discussion and research.

Agitation appears to be a common pheno- menon; therefore, it is essential for neuroscience professionals to have an approach for management. In addition to operationally defining agitation, articles highlight management strategies. This comprehensive review of agitation should be of interst to all rehabilitation and neuroscience professionals who care for brain injured individuals.

In addition to the articles on agitation, this issue of NeuroRehabilitation contains two articles focusing on seizures and their management. These manuscripts were originally part of the special issue on behavioral neurology (Vol. 5, no. 2) prepared by David S. Geldmacher, but appear herein due to space constraints of the previous issue. 